\title{
E²TBR: ENERGY EFFICIENT TRANSMISSION BASED ROUTING FOR IOT NETWORKS
}

\author{
SANTIAGO. $S^{1} \&$ AROCKIAM. $\mathrm{L}^{2}$ \\ ${ }^{I}$ Research Scholar, Department of Computer Science, St. Joseph's College, \\ Autonomous, Tiruchirappalli, Tamil Nadu, India \\ ${ }^{2}$ Associate Professor \& Dean, Department of Computer Science, St. Joseph's College
}

Autonomous, Tiruchirappalli, Tamil Nadu, India

\begin{abstract}
The Internet of Things (IoT) envisions the notion of ubiquitous connectivity of everything. This combines many technologies. Since millions of devices are going to be connected to the internet and wireless in nature, energy is an important factor in IoT. In order to increase the life time of a sensor node, energy must be saved at different levels. This paper proposes Energy Efficient Transmission Based Routing ( $\left.E^{2} T B R\right)$ for IoT Networks. A newer node uses transmission range or coverage for its parent selection in this process. The energy consumption is reduced in comparison with the existing methods.
\end{abstract}

KEYWORDS: Energy Efficiency, Transmission Range, RPL, Routing \& Internet of Things

Received: Jul 04, 2017; Accepted: Jul 24, 2017; Published: Aug 08, 2017; Paper Id.: IJCSEITRAUG201712

\section{INTRODUCTION}

The internet as a set of connected computer devices is changed to Internet of Things (IoT) where every object is connected with every other object. IoT targets to connect various objects with unique addresses, to enable them interact with each other and with the world [1-5].The number of things is larger than the number of world population. The applications include a wide range of domains such as homes, hospital, retail, logistics, industry, agriculture and environmental related issues as shown in figure 1.

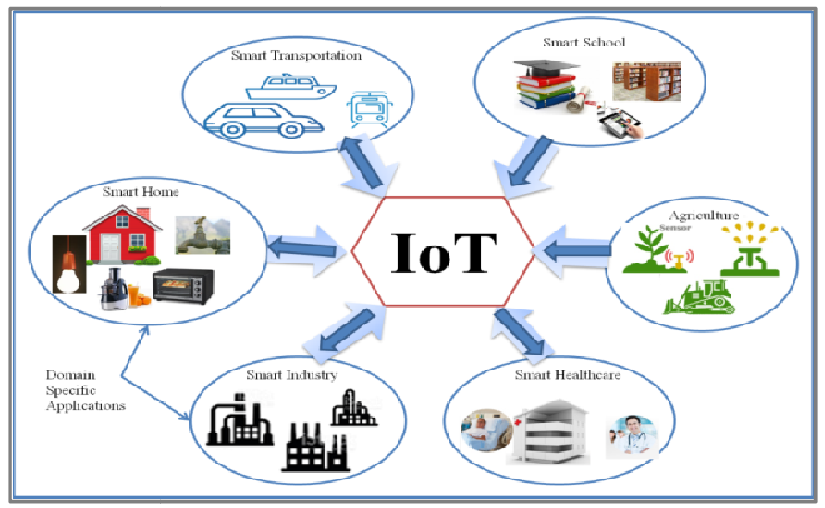

Figure 1: The General Architecture of IoT

To realize this dream, the Internet Engineering Task Force (IETF) Routing Over Low-power and Lossy networks (ROLL) has standardized low power and lossy networks routing architecture called RPL [23]. A key 
feature of RPL is that it represents a specific routing solution for constrained devices.

This paper proposes an Energy Efficient Transmission Based Routing for IoT Networks. The newer node chooses the node which falls at the border line of the transmission range as its parent. In this way, the number of hops is reduced and the delivery ratio is better. This method results in considerable energy saving. Therefore, the life time of the network increases. The rest of this paper is organized in the following manner. Section II presents related works. Section III introduces a brief idea on IoT technology. Section IV explains proposed technique - $\mathrm{E}^{2} \mathrm{TBR}$. The problem definition is presented in section V. The result and discussion are shown in section VI. Finally, section VII concludes this paper.

\section{REVIEW OF LITERATURE}

Mamoun Qasem et al. [6] proposed a new objective function that balanced the number of children nodes for the overburdened nodes to ensure node lifetime maximization. Using this technique, the authors mitigated any potential extra overhead. Vi Chien Thang et al. [7] evaluated the performance of RPL protocol. They proposed an algorithm to choose the optimal path based on two routing metrics such as ETX (Expected Transmission Count) and EI (Energy Indicator) of forwarding nodes.

Santiago et al. [17] presented the issues and ways to minimize the energy consumption in IoT networks. The researchers also highlighted the need for the lightweight algorithms for the resource-constraint devices. Sheeraz et al. [18] designed an enhanced version of RPL for IoMT in which the sensed information was given by the multimedia devices. They also minimized carbon footprint emissions and energy consumption.

Patrick et al. [14] designed an Objective Function for RPL that used node remaining energy as metric. They compared path cost as the minimum node energy level and proposed energy-based path cost. Necip et al. [12] proposed a load balanced network based on parent aware objective function (PAOF). The proposed method used both ETX (Expected Transmission Count) and parent count metrics to compute the best path for routing. The algorithm compared the number of parent counts and selected the minimum one as preferred parent. Fadwa et al. [11] designed bounding degrees on RPL (BD-RPL). They also evaluated BD-RPL using the Cooja simulator and demonstrated improvement over RPL.

Oana et al. [13] first selected two metrics namely MinHop and ETX. Second, chose Link Quality indicator (LQI). They adopted a passive measurement technique. In that, they estimated the Pocket Delivery Ratio using the existing data traffic. Sang et al. [15] suggested EEPR (Energy Efficient probabilistic routing for IoT) algorithm using residual energy of each node and ETX metric in the context of AODV protocol. The proposed algorithm controlled the request packet forwarding process in order to reduce the packet loss and network congestion. They employed two different routing metrics i.e., ETX and residual energy of node. Tao et al. [16] analysed the performance for different network settings and evaluated the behaviour of the Contiki RPL implementation. The evaluated metrics were signaling overhead, latency and energy consumption. The authors concluded that there were several aspects to be improved, like signaling overhead, latency, energy and so on.

Algimantas et al. [8] proposed the energy efficient SSL protocol which ensured the maximum bandwidth and required level of security with minimum energy consumption. They explained the basic concept of the SSL protocol and proposed adaptive SSL protocol. Amol et al. [9] presented the past research work and discussed the applicability towards the IoT. They discussed the existing routing algorithms such as ad-hoc on demand multipath distance vector routing, secure multipath routing protocol, energy aware ant routing, pruned adaptive routing and routing protocol for low power 
and lossy networks. Belghachi et al. [10] used residual energy and transmission delay as routing metrics of the nodes to select the parent. They designed the objective function for this metric based on Ant colony optimization and compared the results of experiments performed with RPL based on ETX.

A couple of designed OF implementations are the Objective Function Zero (OF0) which uses a hop count-based metric [21]. The MRHOF is known as OF1 which selects the path with the smallest ETX value. [22]

\section{TECHNOLOGIES IN IOT}

The IoT system is a network of networks where a massive number of objects/ things/devices are connected through communication technologies. The communication enabling technologies of IoT heavily depend on the connection of objects, collection of data and interaction among the objects. Some of the technologies are RFID, NFC, Bluetooth, WIFi and Zigbee.

RFID stands for Radio-Frequency Identification and is a generic term for technologies that use radio waves to automatically identify people or objects. Near field communication, abbreviated NFC is a form of contactless communication between devices like smart phones or tablets. Contactless communication allows a user to wave the smartphone over a NFC compatible device to send information without needing to touch the devices together.

The first release of Bluetooth was for a wireless data system that could carry data. The aim of Bluetooth technology was to enable users to replace cables between devices. The IEEE 802.11 Wi-Fi standard was released 1999. This is a facility allowing smart objects to connect to the internet or communicate with one another wirelessly within a particular area. There are other enhanced versions. Zigbee is a communication protocol based on IEEE 802.15.4. It supports star, tree and mesh topologies. There are two types of devices namely Full-function devices and reduced function devices. The analysis of technologies is shown in table 1.

Table 1: Communication Technologies for IoT

\begin{tabular}{|c|l|c|c|c|c|}
\hline S No & Technology & Standard & Frequency & Data Bits & Range \\
\hline 1 & RFID & ISO/IEC 1800-6 & $860-960 \mathrm{MHz}$ & $10-100 \mathrm{Kbits} / \mathrm{s}$ & $1-100 \mathrm{~m}$ \\
\hline 2 & NFC & ISO/IEC 18092:2013 & $13.56 \mathrm{MHz}$ ISM & $212,424 \mathrm{Kbits} / \mathrm{s}$ & $20 \mathrm{~cm}$ \\
\hline 3 & Bluetooth & IEEE 802.15 .1 & $2.4 \mathrm{GHz}$ ISM & $1 \mathrm{Mbits} / \mathrm{s}$ & $10 \mathrm{~m}$ \\
\hline 4 & Wi-Fi & IEEE $802.11 \mathrm{n}$ & $2.4 \mathrm{GHz}$ ISM & $54 \mathrm{Mbits} / \mathrm{s}$ & $100 \mathrm{~m}$ \\
\hline 5 & Zigbee & IEEE 802.15 .4 & $2.4 \mathrm{GHz}$ ISM & $250 \mathrm{Kbits} / \mathrm{s}$ & $30 \mathrm{~m}$ \\
\hline
\end{tabular}

\section{THE PROBLEM STATEMENT}

Routing is considered one of the critical issues in IoT due to the large number of devices which are wireless in nature. Energy plays a vital role in extending the lifetime of the network. The RPL protocol standardized by IETF group uses the existing objective functions (Hop Count and ETX) to assign the rank for IoT networks. This traditional method consumes more energy to transfer a packet to the destination.

\section{TRANSMISSION BASED ROUTING}

The proposed technique Energy Efficient Transmission Based Routing for IoT Networks uses transmission range as routing metric of the nodes to assign rank. This metric aims at minimizing the energy conception. The technique is introduced in the OF for calculating rank in RPL Protocol. The method is presented in this paper. 


\subsection{Computing Rank using Objective Function}

The rank is the distance from the node to the DODAG (Destination Oriented Directed Acyclic Graph) in figure 2 [19]. It shows the position of the other nodes with regard to DODAG root. It is a fixed number and the position of the radix point between the integer part and the fractional part determined by Min Hop Rank Increase. Min Hop Rank Increase is the minimum increase in rank between node and any of its DODAG parents. The rank value decreases toward the upward direction and increases in the opposite direction.

- Case 1: When Rank (M) is less than Rank (N), the position of M is closer to the DAG root. Node M is the parent for node $\mathrm{N}$.

- Case 2: When Rank (M) is equal to Rank (N), it may cause a routing loop.

- Case 3: When Rank (M) is more than Rank (N), node M may be in the sub-DODAG of node N. If $\mathrm{N}$ selects node $\mathrm{M}$ as parent, there is a risk of creating a loop. Based on the condition, rank is calculated. If a node $\mathrm{M}$ has chosen its preferred parent $\mathrm{N}$, the rank from preferred parent is

$\operatorname{Rank}(\mathrm{M})=\operatorname{Rank}(\mathrm{N})+1 / \operatorname{Rank}(\mathrm{N})+\mathrm{P}_{\mathrm{MN}}$

Where $\mathrm{P}_{\mathrm{MN}}$ corresponds to the high probability and $\mathrm{M}$ has selected $\mathrm{N}$ as the preferred parent.

The exact calculation of the rank is left to the objective function. Even though rank is left to the OF, it implements generic properties regardless of the OF. In particular, Rank monotonically decreases and it can be considered a location or radius of a node within a DODAG version.

\subsection{Energy Calculation of the Proposed Method}

Let $\mathrm{S}$ be the surface of implementation and $\mathrm{D}_{1}, \mathrm{D}_{2} \ldots \mathrm{D}_{\mathrm{n}}$ are the heterogeneous devices deployed in the surface. The transmission range of each device is denoted by $\mathrm{T}_{1}, \mathrm{~T} 2, \mathrm{Tn}$.

Energy required for a device $\mathrm{D}_{\mathrm{i}}$ is calculated by,

$$
\mathrm{E}_{\text {required }}\left(\mathrm{D}_{\mathrm{i}}\right)=\mathrm{V}\left(\mathrm{I}_{\mathrm{r}} \mathrm{t}_{\mathrm{r}}+\mathrm{I}_{1} \mathrm{t}_{1}+\mathrm{I}_{\mathrm{tr}} \mathrm{t}_{\mathrm{tr}}+\mathrm{I}_{\mathrm{rv}} \mathrm{t}_{\mathrm{rv}}\right)
$$

Where $\mathrm{V}$ is the voltage supplied in the circuit, $\mathrm{I}_{\mathrm{r}}$ is the consumption current of the device while running, $\mathrm{t}_{\mathrm{r}}$ is the time the device has been running, $I_{1}$ and $t_{1}$ are the consumption current and the time of device in low power mode, $I_{t r}$ and $t_{t r}$ are the consumption current and the time of the parent in transmit mode, $\mathrm{I}_{\mathrm{rv}}$ and $\mathrm{t}_{\mathrm{rv}}$ are the consumption current and the time of parent in receive mode.

The residual energy of a node or device $\mathrm{D}_{\mathrm{i}}$ is calculated by

$$
\mathrm{E}_{\text {residual }}\left(\mathrm{D}_{\mathrm{i}}\right)=\mathrm{E}_{\text {initial }}\left(\mathrm{D}_{\mathrm{i}}\right)-\mathrm{E}_{\text {required }}\left(\mathrm{D}_{\mathrm{i}}\right)
$$

The energy indicator of the sensor device is defined by 


$$
\begin{gathered}
\mathrm{E}_{\mathrm{i}}\left(\mathrm{D}_{\mathrm{i}}\right)=\mathrm{\textrm {E } _ { \text { residual } } ( \mathrm { D } _ { \mathrm { i } } )} \\
\mathrm{E}_{\text {initial }}\left(\mathrm{D}_{\mathrm{i}}\right)
\end{gathered}
$$

The range $\mathrm{T}$ of a device is calculated using Cartesian distance between two points $\mathrm{i}$ and $\mathrm{j}$ at $\mathrm{x}_{\mathrm{i}}$ and $\mathrm{x}_{\mathrm{j}}$.

$$
\mathrm{R}_{\mathrm{ij}}=\left\|\mathrm{x}_{\mathrm{i}}-\mathrm{x}_{\mathrm{j}}\right\|=\sqrt{\sum_{k=1}^{d}\left(x_{i, D}-x_{j, D}\right)^{2}}
$$

Where $x_{i}, D$ is the $D^{\text {th }}$ node of the spatial coordinate $x_{i}$ of $i^{\text {th }}$ node.

\subsection{Rank Calculation of the Proposed Method}

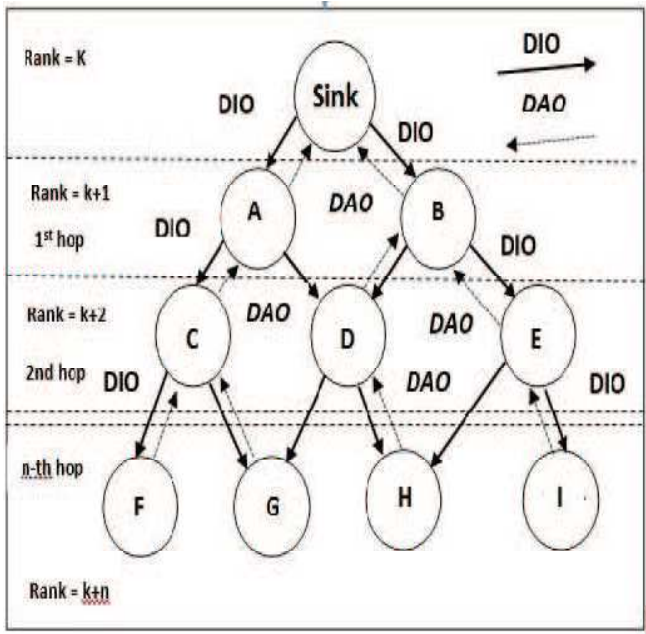

Figure 2: Rank in RPL Network

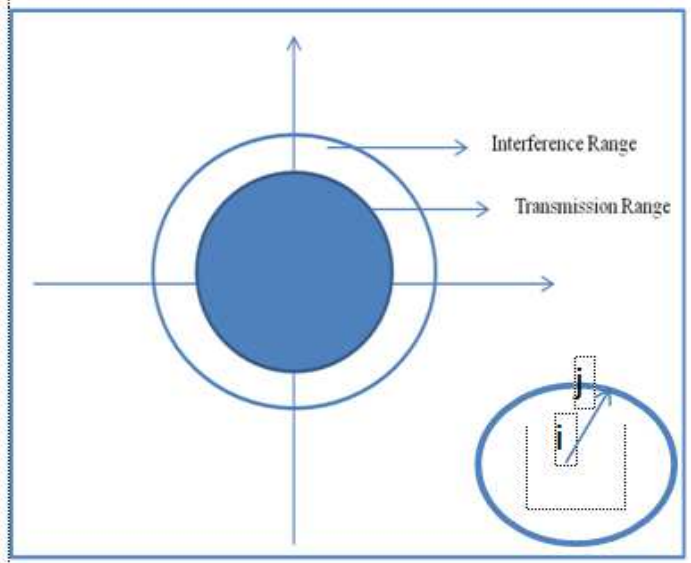

Figure 4: The Proposed Method

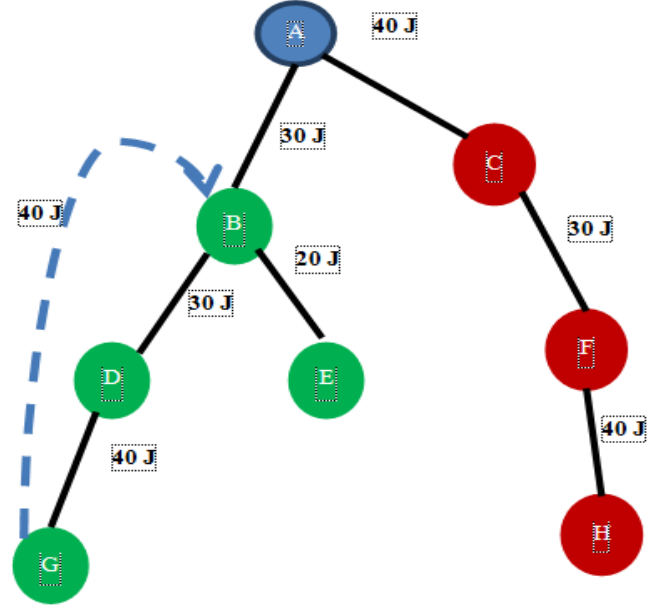

Figure 3: Proposed Rank in RPL

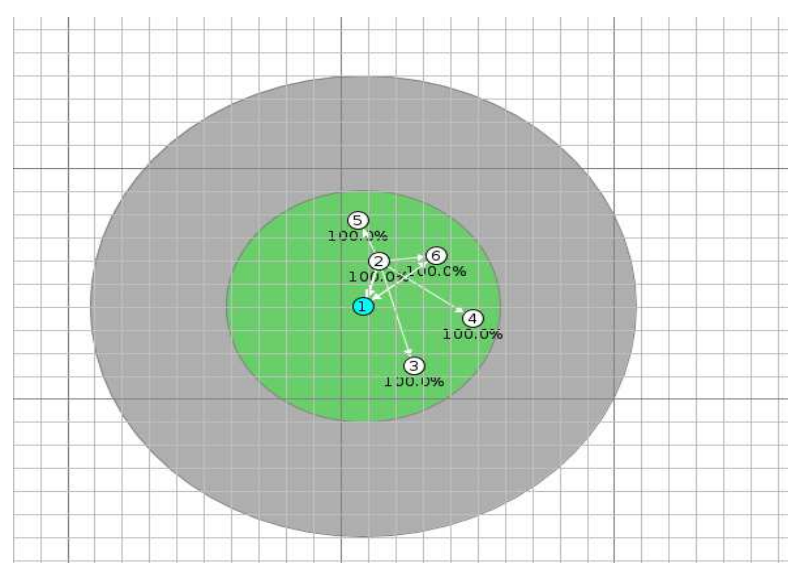

Figure 5: Screen Shot of Proposed Method

ETX and Hop Count are two commonly used routing metrics in the implementation of RPL. Apart from ETX and Hop Count, the proposed transmission based parent selection keeps transmission range as upper limits to select the parent as shown in figure 3. The method is proved using Cartesian distance between two points with the maximum range. The devices within the range of the root will directly communicate to the root as in figure 5 . As in the case of the root node, the devices away from the root will utilize the proposed objective function to choose a parent node as presented in the 
algorithm.

In the proposed model, the data generated by one device will forward the data to another based on transmission range. Due to the diverse applications of IoT, the design of RPL is made flexible to adapt heterogeneous network. The algorithm is used to implement the proposed technique given in Table 2.

Table 2: Algorithm E ${ }^{2}$ TBR for Parent Selection

\begin{tabular}{|l|}
\hline Input: Routing Table of Node $\mathrm{D}_{\mathrm{i}}$ \\
\hline Output: Preferable Parent of Node $\mathrm{D}_{\mathrm{i}}$ \\
\hline Parent_Selected=NULL \\
\hline Input Max_ETX \\
\hline for $\mathrm{i}=1$ to Routing Table $[\mathrm{i}]$ \\
Distance $\left(\mathrm{D}_{\mathrm{i}}\right)=$ ETX $\left(\mathrm{D}_{\mathrm{i}}\right)$. Hop $\left(\mathrm{D}_{\mathrm{i}}\right)$. Tr_range $\left(\mathrm{D}_{\mathrm{i}}\right)$ \\
\hline if $\left(\right.$ Distance $\left(\mathrm{D}_{\mathrm{i}}\right)<$ Max_ETX) then \\
Max_ETX $=$ Distance $\left(\mathrm{D}_{\mathrm{i}}\right)$ \\
Parent_Selected = Distance $\left(\mathrm{D}_{\mathrm{i}}\right)$ \\
end if \\
\hline end for \\
\hline if Parent_Select != NULL then \\
return (Parent_Selected) \\
else \\
send(DIS) \\
end if
\end{tabular}

\section{RESULT AND DISCUSSION}

\subsection{Energy Efficiency}

Nodes within the transmission range of the root node establish the connection and transmission range of the root node is considered as rank 1as shown in figure 5. A new node which is out of the transmission range of the root node sends DIS (DODAG Information Solicitation) messages to solicit DIO (DODAG Information Object) from the neighbours. For the selection of a preferred parent, the newer node uses the maximum transmission range. There are many neighbour nodes for node 18 in figure 6. To transmit data, the newer node chooses the node which is in the upper limits of transmission range as shown in figure $6 a \& b$.

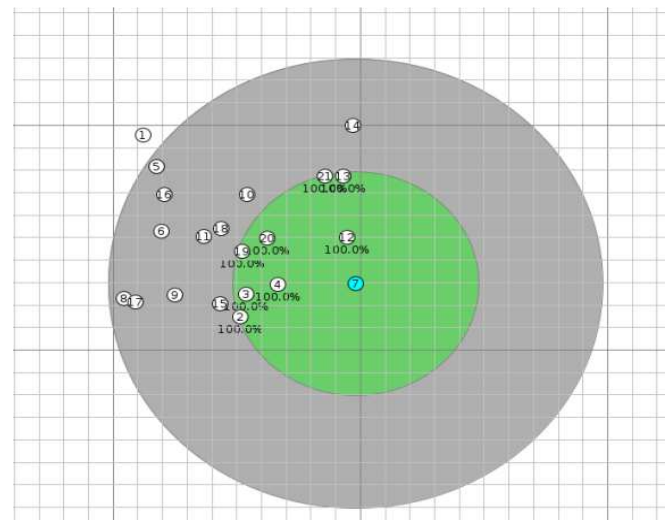

Figure 6 a: Transmission Range of Leaf Node

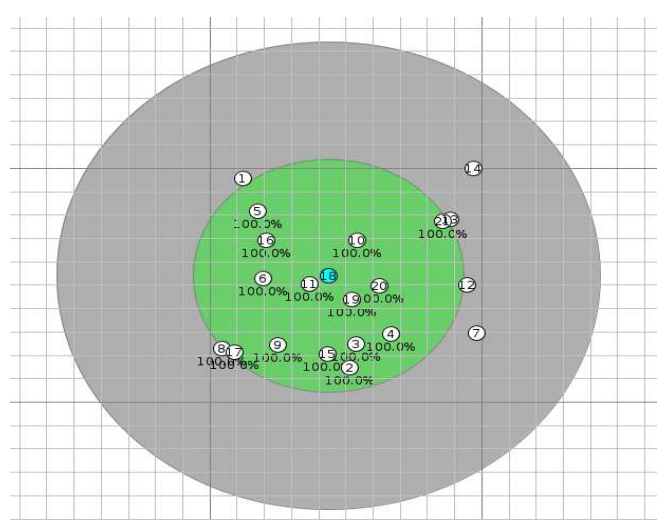

Figure 6 b: Transmission Range of Remote Node

The energy consumed to transfer a packet within the transmission range is same for nodes [19]. There are also other parent nodes which are near having less distance and more energy. But, the newer node chooses the node which falls at the border line of the transmission range. When new node receives the DAO-ACK (Destination Advertisement Object 
Acknowledgement) message with the acceptance, then the route is established and starts sending DAO control messages. In this way, the number of hops are reduced, resulting a considerable energy saving. The delivery ratio is better as the nodes having maximum transmission range leading to better packet ratio. This method reduces transmission delay as the number of hops is reduced.

\subsection{Performance Analysis}

The proposed technique performs better and increases the lifetime of the network. The energy consumption of a single node is given in figure 7. The usual energy consumption for node $\mathrm{G}$ to transfer a packet to the root node is $100 \mathrm{~J}$. Using proposed method, it consumes only $70 \mathrm{~J}$ for a single transaction. The energy consumed for bidirectional transaction is $200 \mathrm{~J}$ for usual operation and $170 \mathrm{~J}$ for the proposed method. The consumption of energy for 10, 50, 100 and 200 nodes is shown in figure 8.

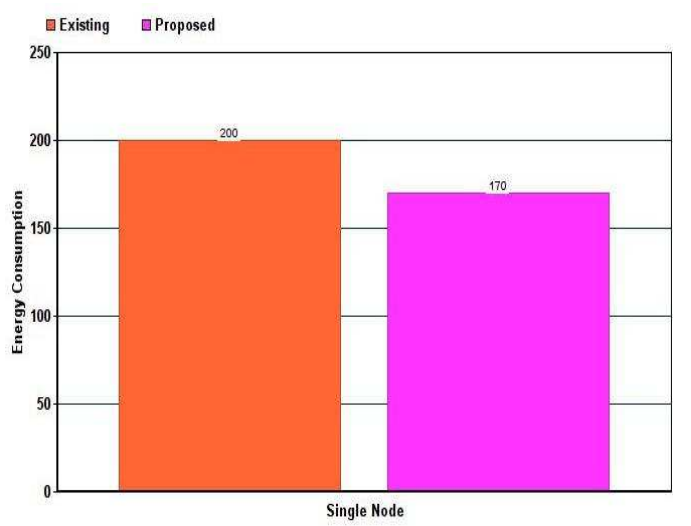

Figure 7: Single Node Energy

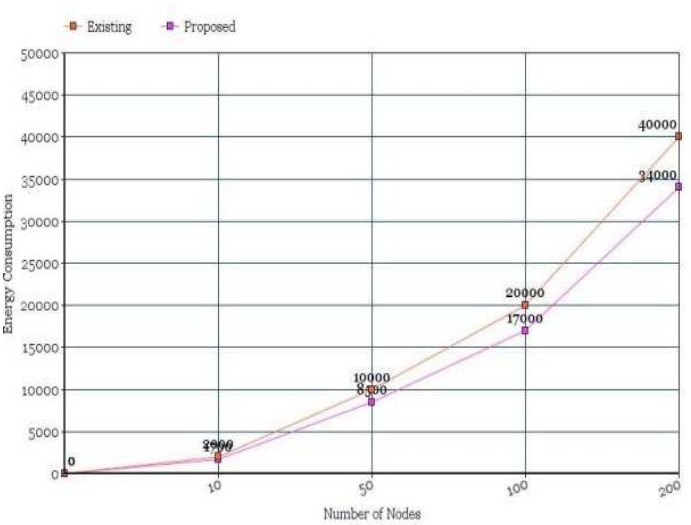

Figure 8: Total Energy Consumption

Where $\mathrm{S}$ is the energy saved per node and $\mathrm{N}$ is the number of nodes. The total Energy is

$\mathrm{TE}=\mathrm{N} . \mathrm{S}$

Total saving increases when number of nodes increases in the network.

\section{CONCLUSIONS}

The internet has changed the life style over last few years. The idea of IoT is finding path in our life, aiming to improve the quality of life by connecting smart devices. This paper suggested transmission based technique using Cartesian distance to enhance routing for IoT networks and increased the life time of the network. The concept is applied to RPL protocol which is mainly designed for constrained smart devices. The performance analysis proves that the number of hops are reduced, resulting a considerable energy saving.

\section{REFERENCES}

1. Perera, Liu, Jayawardena \& Chen. (2014). A survey on Internet of Things from industrial market perspective. IEEE Access, 2, 1660-1679.

2. Da Xu, He \&Li. (2014). Internet of Things in industries: A survey. IEEE Trans. Ind. Information.10, 2233-2243.

3. Perera, Liu \& Jayawardena. (2015).The emerging Internet of Things marketplace from an industrial perspective: A survey. IEEE Transactions on Emerging Topics in Computing, 1-14. 
4. Al-Fuqaha, Guizani, Mohammadi, Aledhari \& Ayyash. (2015). Internet of Things: A survey on enabling technologies, protocols and Applications. IEEE Communication Surveys \&Tutorials, 1, 2347-2374.

5. Shaikh, Zeadally \& Exposito. (2015). Enabling technologies for green Internet of Things. IEEE System Journal, 1-11.

6. Mamoun Qasem, Ahmed Al-Dubai, Imed Romdhani, Baraq, Ghaleb \& Wajeb Gharibi. (2016). A New Efficient Objective Function for Routing in Internet of Things Paradigm. IEEE Standards for Communications and Networking, 1-6.

7. Vi Chien Thang \& Nguyen Van Tao. (2016). A Performance Evaluation of Improved IPv6 Routing Protocol for Wireless Sensor Networks. I.J. Intelligent Systems and Applications, 12, 18-25.

8. Algimantas Venckauskas, Nerijus Jusas, Egidijus Kazanavicius \& Vytautas Stuikys. (2015). An Energy Efficient Protocol for The Internet of Things. Journal of Electrical Engineering, 66, 47-52.

9. Amol Dhumane, Rajesh Prasad \& Jayashree Prasad. (2016). Routing Issues in Internet of Things: A Survey. Lecture Notes in Engineering and Computer Science, 2221, 404-412.

10. Belghachi Mohamed \& Feham Mohamed. (2016). QoS Routing for Low Power and Lossy Networks. International Journal of Distributed Sensor Networks, 11, 1-10.

11. Fadwa Boubekeur, Lélia Blin, Rémy Leone \& Paolo Medagliani. (2015). Bounding Degrees on RPL. Proceedings of the 11th ACM Symposium on QoS and Security for Wireless and Mobile Networks, 123-130.

12. Necip Gozuacik \& Sema Oktug. (2015). Parent-Aware Routing for IoT Networks Chapter: Internet of Things, Smart Spaces, and Next Generation Networks and Systems. 9247 of the series Lecture Notes in Computer Science (Springer), 23-33.

13. Oanalova, Fabrice Theoleyre\& Thomas Noel. (2015). Using multiparent routing in RPL to increase the stability and the lifetime of the network. Ad Hoc Networks, Elsevier, 29, 45-62.

14. Patrick Olivier Kamgueu, Emmanuel Nataf, Thomas DjotioNdie \& Olivier Festor. (2013). Energy-based routing metric for RPL. Research Report-8208, INRIA, 13, 1-14.

15. Sang Hyun Park, Seungryong Cho \&Jung Ryun Lee. (2014). Energy Efficient Probabilistic Routing Algorithm for Internet of Things. Journal of Applied mathematics, 1, 1-7.

16. Tao Zhang \& Xianfeng Li. (2014). Evaluating and Analyzing the performance of RPL in Contiki. ACM Proceedings of the first international workshop on Mobile sensing, computing and communication, 19-24.

17. Santiagoand Arockiam. (2016). Energy Efficiency in Internet of Things: An Overview. International Journal of Recent Trends in Engineering \& Research (IJRTER), 2, 475-482.

18. Sheeraz Alvi, Ghalib shah \&WaqarMahmood. (2015). Energy Efficient Green Routing Protocol for Internet of Multimedia Things. The IEEE International Workshop on Emerging Wireless Sensors Network Applications, 1-6.

19. Abdul Rehman, Meer Muhammad Khan, Ali Lodhi \& Faisal Bashir Hussain. (2016). Rank Attack Using Objection Function in RPL for Low Power and Lossy Networks. Industrial Informatics and Computer Systems, IEEE, 1-5.

20. Jyoti Kaurav \& Kaushik Ghosh. (2012). Effect of Transmitting Radius, Coverage Area and Node Density on the Lifetime of a Wireless Sensor Network. Proc. Int. Conf. on Recent Trends in Information Processing \& Computing. IPC. Elsevier, $110-$ 116.

21. Objective Function Zero for the Routing Protocol for Low-Power and Lossy Networks (RPL). https://tools.ietf.org/pdf/rfc6552

22. The Minimum Rank with Hysteresis Objective Function (RPL). https://tools.ietf.org/html/rfc6719

23. RPL: IPv6 Routing Protocol for Low-Power and Lossy Networks. https://tools.ietf.org/html/rfc6550 